1. Ikeda, F., et al. 2004. Critical roles of c-Jun signaling in regulation of NFAT family and RANKL-regulated osteoclast differentiation. J. Clin. Invest. 114:475-484. doi:10.1172/JCI200419657.

2. Teitelbaum, S.L. 2000. Bone resorption by osteoclasts. Science. 289:1504-1508.

3. Walker, D.G. 1975. Bone resorption restored in osteopetrotic mice by transplants of normal bone marrow and spleen cells. Science. 190:784-785.

4. Udagawa, N., et al. 1990. Origin of osteoclasts: mature monocytes and macrophages are capable of differentiating into osteoclasts under a suitable microenvironment prepared by bone marrow-derived stromal cells. Proc. Natl. Acad. Sci. U. S. A. 87:7260-7264.

5. Lacey, D.L., et al. 1998. Osteoprotegerin ligand is a cytokine that regulates osteoclast differentiation and activation. Cell. 93:165-176.

6. Yasuda, H., et al. 1998. Osteoclast differentiation factor is a ligand for osteoprotegerin/osteoclastogenesis-inhibitory factor and is identical to TRANCE/ RANKL. Proc. Natl. Acad. Sci. U. S. A. 95:3597-3602.

7. Lam, J., Nelson, C.A., Ross, F.P., Teitelbaum, S.L., and Fremont, D.L. 2001. Crystal structure of TRANCE/ RANKL cytokine reveals determinants of receptor-ligand specificity. J. Clin. Invest. 108:971-980.
doi:10.1172/JCI200113890.

8. Lam, J., et al. 2000. TNF- $\alpha$ induces osteoclastogenesis by direct stimulation of macrophages exposed to permissive levels of RANK ligand. J. Clin. Invest. 106:1481-1488.

9. Li, J., et al. 2000. RANK is the intrinsic hematopoietic cell surface receptor that controls osteoclastogenesis and regulation of bone mass and calcium metabolism. Proc. Natl. Acad. Sci. U. S. A. 97:1566-1571.

10. Boyle, W.J., Simonet, W.S., and Lacey, D.L. 2003 Osteoclast differentiation and activation. Nature. 15:337-342.

11. Grigoriadis, A.E., et al. 1994. c-Fos: a key regulator of osteoclast-macrophage lineage determination and bone remodeling. Science. 266:443-448.

12. Lomaga, M.A., et al. 1999. TRAF6 deficiency results in osteopetrosis and defective interleukin-1, CD40, and LPS signaling. Genes Dev. 13:1015-1024.

13. Naito, A., et al. 1999. Severe osteopetrosis, defective interleukin-1 signalling and lymph node organogenesis in TRAF6-deficient mice. Genes Cells. 4:353-362.

14. David, J.-P., Sabapathy, K., Hoffmann, O., Idarraga, M.H., and Wagner, E.F. 2002. JNK1 modulates osteoclastogenesis through both c-Jun phosphorylationdependent and -independent mechanisms. J. Cell. Sci.
115:4317-4325

15. Kenner, L., et al. 2004. Mice lacking JunB are osteopenic due to cell-autonomous osteoblast and osteoclast defects. J. Cell Biol. 164:613-623.

16. Matsuo, K., et al. 2000. Fosl1 is a transcriptional target of c-Fos during osteoclast differentiation. Nat. Genet. 24:184-187.

17. Takayanagi, H., et al. 2002. Induction and activation of the transcription factor NFATc1 (NFAT2) integrate RANKL signaling in terminal differentiation of osteoclasts. Dev. Cell. 3:889-901.

18. Macian, F., Lopez-Rodriguez, C., and Rao, A. 2001. Partners in transcription: NFAT and AP-1. Oncogene. 20:2476-2489.

19. Zhou, B., et al. 2002. Regulation of the Murine Nfatc1 Gene by NFATc2. J. Biol. Chem. 277:10704-10711.

20. Srivastava, S., et al. 2000. Estrogen decreases osteoclast formation by down-regulating receptor activator of NF-kappa B ligand (RANKL)-induced JNK activation. J. Biol. Chem. 276:8836-8840. doi:10.1074/jbc.M010764200.

21. Eghbali-Fatourechi, G., et al. 2003. Role of RANK ligand in mediating increased bone resorption in early postmenopausal women. J. Clin. Invest. 111:1221-1230. doi:10.1172/JCI200317215.

\title{
AMP-activated protein kinase: the guardian of cardiac energy status
}

\author{
D. Grahame Hardie
}

Division of Molecular Physiology, Faculty of Life Sciences, Wellcome Trust Biocentre, University of Dundee, Dundee, United Kingdom.

\begin{abstract}
Several years ago it was proposed that the AMP-activated protein kinase cascade might protect cells against stresses that deplete cellular ATP. Young et al. have now directly tested this by studying the effects of ischemia and reperfusion in perfused hearts from mice expressing a dominant-negative mutant that suppresses the kinase activity in cardiac muscle (see the related article beginning on page 495). Compared with control hearts, the mutant hearts showed clear evidence for increased necrotic damage and increased apoptosis. These findings may have implications for the treatment of ischemic heart disease.
\end{abstract}

AMP-activated protein kinase (AMPK) is the downstream component of a protein kinase cascade that is highly conserved in all eukaryotic cells (1). AMPK is activated by the rising cellular AMP that (due to the action of adenylate kinase) always $\mathrm{ADP}$ ratio, and this activation is antagonized by high concentrations of ATP (Figure 1). Downstream targets and processes regulated by the kinase are being identified on a regular basis (Figure 2).

Nonstandard abbreviations used: 5-aminoimidazole4-carboxamide riboside monophosphate (ZMP); AMPactivated protein kinase (AMPK).

Conflict of interest: The author has declared that no conflict of interest exists.

Citation for this article:

J. Clin. Invest. 114:465-468 (2004).

doi:10.1172/JCI200422683. accompanies a fall in the cellular ATP/
In general, AMPK switches off ATP-consuming processes such as biosynthetic pathways, while switching on catabolic processes that generate ATP, including cellular uptake of glucose (2) and fatty acids (3) and increased fatty acid oxidation (4) in the heart.

The first evidence that AMPK was activated by metabolic stresses appeared 13 years ago, when my group found that AMPK was activated by ATP depletion caused by incubation of isolated rat hepatocytes with high fructose (5), while Witters et al. (6) reported that it was activated by various metabolic poisons in hepatoma cells. We proposed at the time

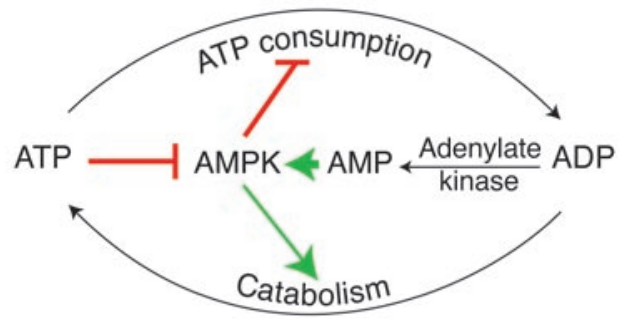

Figure 1

Role of AMPK in regulating energy balance at the single-cell level. The way in which the AMPK system controls the balance between ATP consumption (e.g., by biosynthesis, cell growth, or muscle contraction) and ATP production via catabolism is illustrated. If the rate of ATP consumption exceeds its rate of production, ADP will tend to rise and be converted to AMP by the enzyme adenylate kinase. The rise in level of the activating ligand AMP, coupled with the fall in level of the inhibitory nucleotide ATP, activates AMPK, which then switches off ATP-consuming processes and switches on catabolism in an attempt to redress the balance. 

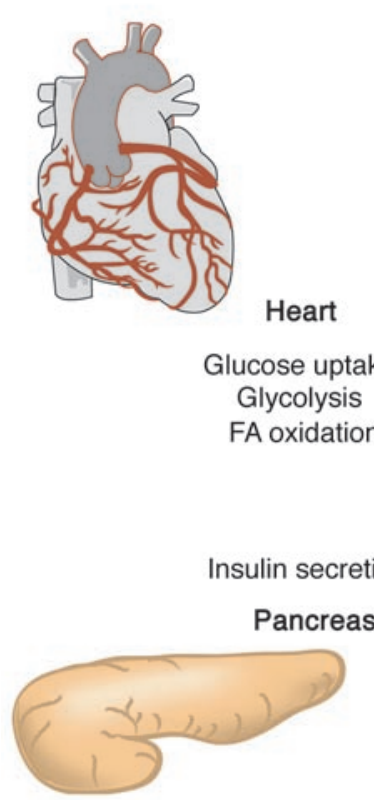

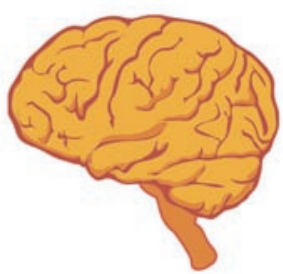

Hypothalamus Food intake

\section{ke}

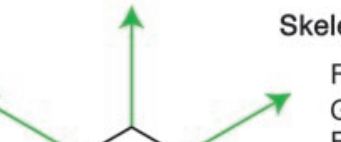<smiles>C1CCCC1</smiles><smiles>CC1CCC(C)C1CO</smiles>
FA synthesis Cholesterol synthesis Gluconeogenesis

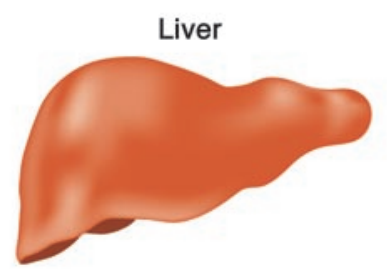

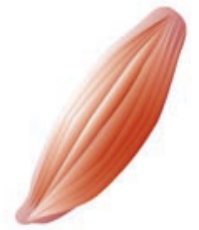

keletal muscle

FA oxidation Glucose uptake Expression of GLUT4 Mitochondria

FA synthesis Lipolysis

Adipose tissue

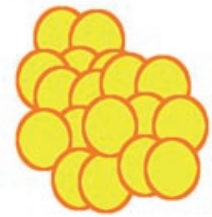

that the AMPK cascade was a system that might protect cells against stresses that compromise their energy status. Ischemia was one obvious such stress, and Lopaschuk et al. later reported that the kinase was activated by ischemia in perfused rat hearts and that this was associated with high rates of fatty acid oxidation during reperfusion (4). Subsequently, Young et al. provided evidence that AMPK activation in the heart increased glucose uptake via translocation of the transporter GLUT4 to the plasma membrane (2), while Hue's group found that this activation caused phosphorylation and activation of the cardiac isoform of 6-phosphofructo-2-kinase, leading to an increase in fructose-2,6-bisphosphate, an activator of glycolysis (7). By these mechanisms, activation of AMPK during periods when the blood supply was compromised during ischemic heart disease would stimulate both glucose uptake and glycolysis and thus generate more ATP via anaerobic metabolism, while high rates of fatty acid oxidation would replenish ATP during subsequent reperfusion. Taken together, these findings suggested that AMPK might provide protection during ischemia and reperfusion. This remained, however, only an attractive hypothesis until now, with a new study by Young and coworkers that is reported in this issue of the JCI (8).

\section{Mouse hearts with reduced AMPK are more susceptible to damage during ischemia and reperfusion}

Previously, Birnbaum's group had constructed transgenic mice that expressed an inactive mutant of the $\alpha 2$ isoform of the AMPK catalytic subunit from a muscle creatine kinase promoter, which provided evidence that AMPK regulated glucose uptake in skeletal muscle in vivo (9). AMPK is a heterotrimer containing, in addition to the catalytic $\alpha$ subunit, a $\beta$ subunit with a glycogen-binding domain $(10,11)$ and a $\gamma$ subunit with two regulatory sites that bind AMP and ATP antagonistically (12). Birnbaum (9) had shown that expression of inactive $\alpha 2$ was very effective at suppressing endogenous AMPK activity and thus behaved as a dominant-negative mutant. This probably works because the inactive $\alpha 2$ competes with the active, endogenous $\alpha 1$ and $\alpha 2$ subunits for binding to the available $\beta$

\section{Figure 2}

Role of AMPK in regulating energy balance at the whole-body level. Green arrows indicate positive effects, and red lines with bars indicate negative effects. In the hypothalamus, activation of AMPK in response to low glucose or leptin levels increases food intake $(18,19)$; references for other effects of AMPK activation can be found in recent reviews (1). FA, fatty acid.

and $\gamma$ subunits and (because the muscle creatine kinase promoter provides very high expression levels) completely displaces them from cellular AMPK complexes (Figure 3).

Because the creatine kinase promoter is also active in cardiac muscle, Young and coworkers were able to use Birnbaum's mouse model to study the role of AMPK in the heart (8). They confirmed that $\alpha 2$ activity was almost totally absent in the heart, although, surprisingly, the basal activity of the $\alpha 1$ isoform was not reduced. This would have been expected, because the $\alpha 1$ isoform also needs to bind to the $\beta$ and $\gamma$ subunits, but the authors suggest that the retention of $\alpha 1$ activity might result from the fact that much of the $\alpha 1$ isoform being measured is actually expressed in cells other than cardiac myocytes, such as endothelial cells. The hearts from the transgenic mice did not display any gross structural abnormalities, although they were marginally smaller than hearts from wild-type littermates, had a lower glycogen content, and exhibited a modest impairment in some parameters of contractile function. Of course, these mice also have abnormalities in skeletal muscle and have been found to take less voluntary exercise (9), so some of the changes in the heart could be secondary to defects in skeletal muscle. A previous study, which did not examine cell survival after ischemia and reperfusion, got around this problem by expressing the inactive $\alpha 2$ from a cardiacspecific promoter (13). However, Young et al. observed the most interesting changes in cardiac function, which are not likely to be secondary effects, using isolated perfused hearts. During low-flow ischemia in hearts of wild-type littermates, the activities of the $\alpha 1$ and $\alpha 2$ isoforms of AMPK increased markedly, as expected, and both remained elevated during reperfusion. However, in the transgenic hearts, $\alpha 2$ activity was negligible under all conditions, while the $\alpha 1$ activity did not 


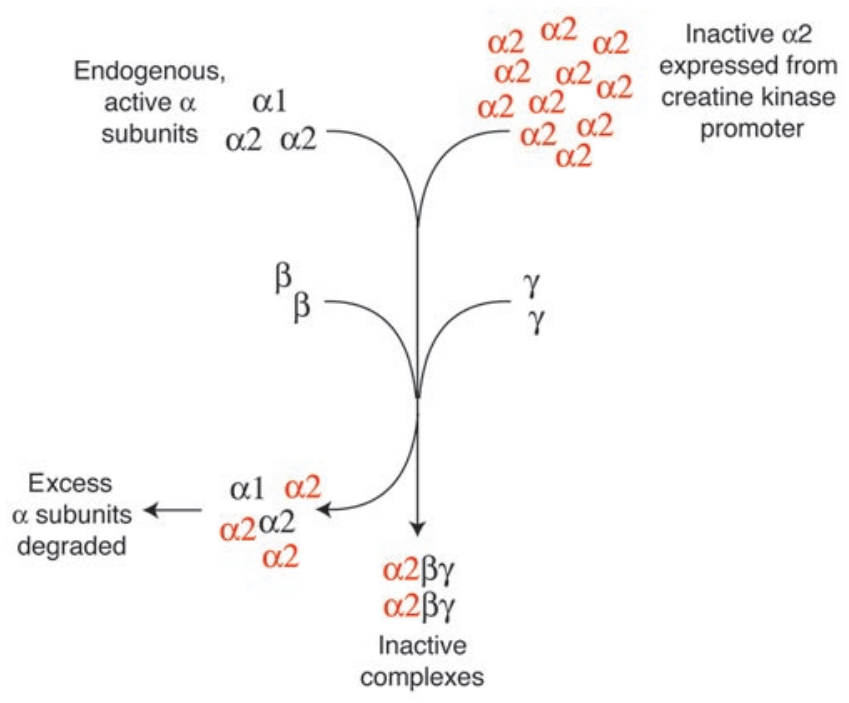

increase above basal values. Satisfyingly, the altered metabolic responses of the hearts from the transgenic animals were completely consistent with the proposed roles of AMPK in controlling cardiac metabolism. Thus, the normal increases in glucose uptake and lactate production during ischemia and reperfusion, and the increase in fatty acid oxidation during reperfusion, were all abolished. The ATP and phosphocreatine contents were also lower in the transgenic hearts during ischemia and did not show any signs of recovery during reperfusion. In addition, left-ventricular contractile function was poorer in the transgenic hearts during ischemia, and recovery during reperfusion was impaired. Most interesting of all, this was accompanied by evidence of both increased necrotic tissue damage and increased apoptosis. Thus, AMPK protects against injury caused by ischemia and reperfusion, most likely by helping to preserve the levels of cellular ATP.

\section{Could AMPK activation be used to treat ischemic heart disease?}

While many in the AMPK field have long held the view, almost as an article of faith, that the kinase protects cells against the damaging effects of metabolic insults such as ischemia, the article by Young and colleagues (8) is important because it provides the first direct evidence that this is indeed the case. Could drugs that activate AMPK provide protection against tissue damage caused by ischemia and reperfusion? Evidence from trials using the drug acadesine (5-aminoimidazole4-carboxamide riboside, or AICAR) sug- gests that this is at least a possibility (14). Acadesine is an adenosine analog that is taken up into cells via adenosine transporters (15) and converted inside the cell by adenosine kinase to 5-aminoimidazole-4-carboxamide riboside monophosphate (ZMP), an intermediate in the synthesis of GTP and ATP. It was originally proposed that acadesine might have beneficial effects by replacing, via de novo synthesis, the depleted levels of these nucleotides caused by ischemia and reperfusion. However, what was not realized at that time was that $\mathrm{ZMP}$ also mimics the effects of AMP to activate the AMPK system (16). In studies using animal models, acadesine has been shown to provide protection against injury caused by ischemia and reperfusion, and results in trials during human coronary artery bypass graft surgery have shown modest benefits (14). The rather limited efficacy of acadesine in these trials could either have been because the drug is not a very potent activator of AMPK (especially in cardiac myocytes; ref. 17), or because the AMPK system would have been rapidly activated during ischemia anyway and did not need a helping hand. However, further studies with novel, more potent AMPK activators are clearly warranted, and with the help of new insights such as those provided by the article in this issue (8), it is possible that this approach might produce new treatments for ischemic heart disease.

\section{Acknowledgments}

Work in the author's laboratory is supported by the Wellcome Trust and the

\section{Figure 3}

How the dominant-negative mutant works. AMPK is an $\alpha \beta \gamma$ heterotrimer, and the catalytic $\alpha$ subunit is not functional unless it is complexed with $\beta$ and $\gamma$. Expression in cardiac myocytes of the inactive, mutant $\alpha 2$ subunit (red) from a strong promoter (muscle creatine kinase) that gives high expression levels means that expression of the inactive $\alpha 2$ is much higher than that of the endogenous, active $\alpha 1$ and $\alpha 2$ subunits. Because the availability of the $\beta$ and $\gamma$ subunits is limiting, most of the heterotrimers formed will contain the inactive, mutant $\alpha 2$. The excess $\alpha$ subunits (not complexed with $\beta$ and $\gamma$ ) are probably recognized by cellular machinery as misfolded proteins and degraded.

European Commission (contract no. QLG1-CT-2001-01488).

Address correspondence to: D. Grahame Hardie, Division of Molecular Physiology, Faculty of Life Sciences, Wellcome Trust Biocentre, University of Dundee, Dow Street, Dundee, DD1 5EH, Scotland, United Kingdom. Phone: 44-1382344253; Fax: 44-1382-345783; E-mail: d.g.hardie@dundee.ac.uk.

1. Hardie, D.G., Scott, J.W., Pan, D.A., and Hudson, E.R. 2003. Management of cellular energy by the AMP-activated protein kinase system. FEBS Lett. 546:113-120.

2. Russell, R.R., Bergeron, R., Shulman, G.I., and Young, L.H. 1999. Translocation of myocardial GLUT-4 and increased glucose uptake through activation of AMPK by AICAR. Am. J. Physiol. 277:H643-H649.

3. Luiken, J.J., et al. 2003. Contraction-induced fatty acid translocase/CD36 translocation in rat cardiac myocytes is mediated through AMP-activated protein kinase signaling. Diabetes. 52:1627-1634.

4. Kudo, N., Barr, A.J., Barr, R.L., Desai, S., and Lopaschuk, G.D. 1995. High rates of fatty acid oxidation during reperfusion of ischemic hearts are associated with a decrease in malonyl-CoA levels due to an increase in $5^{\prime}$-AMP-activated protein kinase inhibition of acetyl-CoA carboxylase. J. Biol. Chem. 270:17513-17520.

5. Moore, F., Weekes, J., and Hardie, D.G. 1991. AMP triggers phosphorylation as well as direct allosteric activation of rat liver AMP-activated protein kinase. A sensitive mechanism to protect the cell against ATP depletion. Eur. J. Biochem. 199:691-697.

6. Witters, L.A., Nordlund, A.C., and Marshall, L. 1991. Regulation of intracellular acetyl-CoA carboxylase by ATP depletors mimics the action of the 5 '-AMP-activated protein kinase. Biochem. Biophys. Res. Commun. 181:1486-1492.

7. Marsin, A.S., et al. 2000. Phosphorylation and activation of heart PFK-2 by AMPK has a role in the stimulation of glycolysis during ischaemia. Curr. Biol. 10:1247-1255.

8. Russell, R.R., III, et al. 2004. AMP-activated protein kinase mediates ischemic glucose uptake and prevents postischemic cardiac dysfunction, apoptosis, 
and injury. J. Clin. Invest. 114:495-503. doi:10.1172/ JCI200419297.

9. Mu, J., Brozinick, J.T., Valladares, O., Bucan, M., and Birnbaum, M.J. 2001. A role for AMP-activated protein kinase in contraction- and hypoxia-regulated glucose transport in skeletal muscle. Mol. Cell. 7:1085-1094.

10. Hudson, E.R., et al. 2003. A novel domain in AMPactivated protein kinase causes glycogen storage bodies similar to those seen in hereditary cardiac arrhythmias. Curr. Biol. 13:861-866.

11. Polekhina, G., et al. 2003. AMPK b-Subunit targets metabolic stress-sensing to glycogen. Curr. Biol. 13:867-871.

12. Scott, J.W., et al. 2004. CBS domains form energysensing modules whose binding of adenosine ligands is disrupted by disease mutations. J. Clin.
Invest. 113:274-284. doi:10.1172/JCI200419874.

13. Xing, Y., et al. 2003. Glucose metabolism and energy homeostasis in mouse hearts overexpressing dominant negative alpha2 subunit of AMP-activated protein kinase. J. Biol. Chem. 278:28372-28377.

14. Mangano, D.T. 1997. Effects of acadesine on myocardial infarction, stroke, and death following surgery. A meta-analysis of the 5 international randomized trials. The Multicenter Study of Perioperative Ischemia (McSPI) Research Group. JAMA. 277:325-332.

15. Gadalla, A.E., et al. 2004. Distinct mechanisms underlie the activation of rat brain AMP-activated protein kinase and the inhibition of excitatory synaptic transmission by AICA riboside (Acadesine) in area CA1 of rat hippocampus. J. Neurochem. 88:1272-1282.

16. Corton, J.M., Gillespie, J.G., Hawley, S.A., and Har- die, D.G. 1995. 5-Aminoimidazole-4-carboxamide ribonucleoside: a specific method for activating AMP-activated protein kinase in intact cells? Eur. J. Biochem. 229:558-565.

17. Javaux, F., Vincent, M.F., Wagner, D.R., and van den Berghe, G. 1995. Cell-type specificity of inhibition of glycolysis by 5-amino-4-imidazolecarboxamide riboside. Lack of effect in rabbit cardiomyocytes and human erythrocytes, and inhibition in FTO2B rat hepatoma cells. Biochem. J. 305:913-919.

18. Andersson, U., et al. 2004. AMP-activated protein kinase plays a role in the control of food intake. J. Biol. Chem. 279:12005-12008.

19. Minokoshi, Y., et al. 2004. AMP-kinase regulates food intake by responding to hormonal and nutrient signals in the hypothalamus. Nature. 428:569-574

\title{
Immune recognition of self in immunity against cancer
}

\author{
Alan N. Houghton and José A. Guevara-Patiño
}

The Swim Across America Laboratory of Tumor Immunology, Memorial Sloan-Kettering Cancer Center, New York, New York, USA.

\begin{abstract}
Most antigens expressed by human cancer cells and recognized by host $T$ cells and antibodies are nonmutated self antigens - molecules also expressed on the surface of normal cells. These self antigens are ineffective at triggering immune responses against cancer cells, which provides one explanation for the difficulties in trying to immunize against human cancer. A new study describes how tumors can avoid recognition by the immune system and how enhancing the affinity of the interaction between a self antigen and the MHC-I molecule may lead to cancer immunity (see the article beginning on page 551).
\end{abstract}

\section{The self/nonself paradigm}

Immunologists generally agree that the immune system was shaped through evolution by the necessity of discriminating nonself pathogens from self tissues (1). This distinction is extremely important for the survival of multicellular organisms. Some strategies for the recognition of nonself pathogens are clear-cut. For example, receptors such as toll-like receptors on host leukocytes bind to certain bacterial or fungal molecules, leading to mobilization of host defenses by signaling the synthesis of molecules that initiate innate and adaptive immune responses. The adaptive immune system, with its diversity of $\mathrm{T}$ cell receptors (TCRs) and antibodies, uses even more precise mechanisms for the recognition of nonself pathogens. This view of self/nonself

Nonstandard abbreviations used: $T$ cell receptor (TCR).

Conflict of interest: The authors have declared that no conflict of interest exists.

Citation for this article:

J. Clin. Invest. 114:468-471 (2004).

doi:10.1172/JCI200422685 provides a logical and useful description of how the immune system responds to exogenous microbes that cause disease.

\section{Recognition of cancer by the host's immune system}

Understanding and achieving immunity to cancer does not fit neatly into the self/ nonself paradigm because cancer is not an exogenous pathogen, but rather arises from normal host cells. In this regard, cancer antigens recognized by the human immune system are self or mutated self molecules (2). Explaining the genetic basis for the pathogenesis of cancer is necessary to understanding the difficulties that the immune system has in recognizing cancer cells.

Protooncogenes and tumor suppressor genes are normal cellular genes that control crucial cell functions, particularly growth and survival (3). Mutations in these genes lead to the emergence of cancer. In addition, loss of expression of tumor suppressor genes contributes to malignant transformation through major deletions in their genetic sequences or silencing by methylation of nucleotides in promoter regions that control gene expression. These deleted or silent genes are incapable of producing antigenic targets for the immune system, but mutated protooncogenes, tumor suppressor genes, or other self genes might generate mutant protein products that could serve as suitable antigens.

An accumulation of genetic alterations in protooncogenes and tumor suppressor genes leads to profound changes in normal cells, including immortality, a block in terminal differentiation, an ability to invade normal tissues and recruit new blood vessels, and the potential to metastasize to distant organs (3). However, because the immune system is trained not to respond to self molecules (in order to avoid autoimmunity), antigenic changes in malignant cells that are created by individual mutations can be rather subtle from the standpoint of the immune system - cancer cells still utilize essentially the same cellular molecules as healthy cells to regulate growth and survival (3). Perhaps mutations in self proteins can be viewed as generating nonself proteins, comparable to foreign proteins from pathogens or other species. This argument has been used as the basis for conducting experiments in mice for the study of tumor immunity using tumors expressing viral or chicken proteins.

$\mathrm{T}$ cells, which are immune cells crucial for rejecting tumors, use their TCRs to recognize short antigenic peptides bound 